\title{
The use of laser dynamical speckle interferometry in the study of cellular processes
}

\author{
A. P. Vladimirov ${ }^{1,2,3}$, I. A. Novoselova ${ }^{1 *}$, Y. A. Mikhailova ${ }^{1,2}$, A. A. Bakharev ${ }^{2}$, and D. I. Yakin ${ }^{1}$ \\ ${ }^{1}$ Ural Federal University named after the first President of Russia Boris N. Eltsin, Ekaterinburg, Russia \\ ${ }^{2}$ Ekaterinburg Research Institute of Virus Infections, Ekaterinburg, Russia \\ ${ }^{3}$ Institute of Engineering Science, Urals Branch, Russian Academy of Sciences, Ekaterinburg, Russia \\ *e-mail: inovoselova93@gmail.com
}

\begin{abstract}
A speckle interferometry setup is mounted, tested and approved that allows the analysis of intracellular processes with large optical magnification. For different part of the cell, the dependences of speckle image correlation coefficients upon time are found. It is shown that the method allows the detection of differences in the process that occur in the nutrient solution outside the cell and inside the cell, as well as those occurring in different parts of the cell. (C) 2016 Journal of Biomedical Photonics \& Engineering.
\end{abstract}

Keywords: laser, speckle, speckle interferometry, cell metabolism, correlation.

Paper \#2952 received 2016.01.31; revised manuscript received 2016.02.12; accepted for publication 2016.03.03; published online 2016.03.30. doi: 10.18287/JBPE16.02.010302

\section{References}

1. M. Francon, Laser Speckle and Applications in Optics, Academic Press, New York (1979). ISBN: 9780124337459.

2. V. V. Tuchin, Lasers and Optical Firbes in Biomedical Studies, 2nd ed., Fizmatlit, Moscow (2010) [in Russian].

3. V. P. Tychinskii, "Coherent phase microscopy of intracellular processes," Physics-Uspekhi 44(6), 617-629 (2001).

4. D. A. Zimnyakov, V. V. Tuchin, and S. R. Utz, "A study of statistical properties of partially developed speckle fields as applied to the diagnostics of structural changes in human skin," Opt. Spectrosc. 76, 838-844 (1994).

5. A. P. Vladimirov, A. L. Lisin, V. I. Mikushin, M. M. Kokhan, I. A. Kuklin, and E. V. Kononenko, "Biological activity of the human skin studied by method of speckle counting," Technical Physics Letters 26(5), 366-368 (2000).

6. G. G. Akchurin, V. V. Bakutkin, E. Y. Radchenko, and V. V. Tuchin, "Laser speckle interferometry and the possibility to determine the retinal acuity of vision in the case of cataract," Biomeditsinskiye tekhnologii $\mathrm{i}$ radioelektronika 1, 19-27 (2002) [in Russian].

7. Y. N. Dubnishchev, V. A. Arbuzov, and P. Y. Belousov, Optical Methods of Flow Studies, Novosibirsk ed., Sibirskoye universitetskoye izd-vo, Novosibirsk (2003) [in Russian].

8. A. P. Poryvaeva, and T. S. Nekrasova, "Cell cultures in the complex diagnostics of herpes virus infections," 6ya regional'naya nauchno-prakticheskaya konferentsiya "Perpektivy ispol'zovaniya v meditsinskoy praktike novykh immunobiologicheskikh preparatov, poluchennykh na osnove kletochnykh struktur," May 30-31, Ekaterinburg, Sbornik materialov (2012).

9. V. V. Lychagov, A. L. Kalyanov, D. V. Lyakin, and V. P. Ryabukho, "Method for remote diagnostics of the internal structure of layered media," Quantum Electron. 38(6), 563-569 (2008).

10. A. P. Vladimirov, A. V. Druzhinin, A. S. Malygin, and K. N. Mikitas, "Theory and calibration of speckle dynamics of phase object," Proc. of SPIE 8337, 83370C (2012).

11. A. S. Malygin, N. V. Bebenina, A. P. Vladimirov, K. N. Mikitas, and A. A. Bakharev, "A speckleinterferometric device for studying the cell biological activity," Instruments and Experimental Techniques 55(3), 415-418 (2012).

12. N. V. Bebenina, "Application of dynamic speckle interferometry method to the study of metabolic activity of cells in the in vitro experiment," Master Thesis, Ekaterinburg (2012) [in Russian]. 
13. Y. A. Mikhailova, "Application of dynamic speckle interferometry to the analysis of metabolic activity of cultivated cells," graduate work, Ekaterinburg (2013) [in Russian].

14. N. P. Glinskikh, V. N. Avdeeva, F. Y. Zusman, et al., Patent No. 2148644 of Russian Federation.

\section{Introduction}

The progress in cell technologies makes it particularly important to develop the method of noninvasive control of the cell physiological condition. For this aim, the indirect techniques are mainly applied, based on the examination of the products discharged from the cell in the course of metabolism, which is not always adequate to the real cell vital activity. At present, there are a few basic methods of cell investigation, e.g., light microscopy, phase-contrast microscopy, fluorescence microscopy, electron microscopy, autoradiography, centrifuging, freezing-spalling techniques. All these methods are rather labour and time consuming, require careful preparation of samples, which makes the fullscale diagnostics of living cells unpractical.

Promising for studying the living cell activity are the optical coherence methods based on the use of lasers. The laser radiation of moderate intensity exerts nonspecific thermal action on biological objects, while the high-intensity laser radiation destroys the biological objects, manifesting itself like other electromagnetic radiations of different nature. However, at relatively low intensities it appears possible to use light for studying the processes in a living object without introducing any serious disturbance of its behaviour. The region of extremely small intensities is of interest, in which it is possible to apply a number of most sensitive methods that do not require strong light fluxes and, therefore, do not introduce distortions into the measurement results due to the homeostasis of living matter even at the local level.

It follows that the extension of the use of optical methods, including the coherence optical ones, in the control of the object condition and diagnostics is caused by the availability, high speed, clearness of information, with the possibility to provide high accuracy of the measurements. Besides the comparability of the optical wavelength with the elements of the controlled object microstructure and the interference of multiple waves allow the possibility to study the object structures at the microscopic level.

In the present paper in order to study the micro- and macroscopic processes that occur in cells, we apply the method of recording the dynamics of laser speckles, or biospeckles. At present, the biospeckles are successfully used to study the activity of different biological objects [1-8]. Earlier the dynamics of speckles was already used to estimate the biological activity of seeds, human skin, and other objects. One of the most successful examples of using the biospeckles is the development of the approach, in which by the contrast of the speckles the blood flow velocity at the eye retina and in near-surface cutaneous coverings of a patient's limb were assessed. At present, the method is well developed both theoretically and experimentally. In Ref. [9] the method of autocorrelation low-coherence interferometry is considered for using in the studies of optical inhomogeneities and internal structure of layered media of technical and biological origin that allows the study of layered objects, among which a cell can be considered, but this method is also capable of analysing the structure and the shape of an object. The present paper is aimed at the study of the processes in real time that occur in the cell membranes and in the cells themselves, which is an urgent problem from both the academic and the practical point of view.

The urgency of such studies is due to the fact that human diseases are directly or indirectly related to the failure of normal functioning of cells or biological membranes. Many diseases are caused by modifications of the structure and functional properties of the cell molecular components under the action of either the external factors or the internal disorders.

In Ref. [10] the theoretical justification of the method was given and the calibration was implemented. In Ref. [11] the parameter characterising the metabolic activity of cells was chosen and justified. The authors of Ref. [12] demonstrated the possibility to study the interaction of herpes with cell cultures and in Ref. [13] the technique was upgraded to obtain stable and reproducible results.

In the above papers, the determination of the parameter characterising the cell metabolic activity was implemented by evaluating the degree of correlation between two speckle patterns. The correlation of speckle patterns was recorded in two areas. The first area was chosen in the image of cells and the second one beyond this image. The technique was developed that allowed one to determine the quantity characterising the cell metabolic activity from two values of the degree of correlation of the image areas. It was shown that such quantity is the root-mean-square deviation of the optical path difference for the pairs of waves, probing the cell.

In all above papers, a few cells could be placed in the area, the linear size of which equals the lens linear resolution. The determination of the parameter characterising the metabolic activity was implemented by averaging over hundreds of cells. However, the logics of the method development required the investigation of the phenomena that occur within a single cell near the membrane, in the cytoplasm or the nucleus.

The aim of this work was to create a speckle interferometric setup for the analysis of intracellular processes under high optical magnification, as well as the study of the change of the parameter characterising the degree of correlation of two speckle patterns, recorded inside and outside individual cells. 
As compared to the analogues, the main advantage of the setup is its high sensitivity. The existing methods and instruments use the double beam interference of waves, and in our system the interferometry of multiple waves is implemented, therefore the sensitivity of the method is by 1-2 orders of magnitude higher than in the analogues. This offers new possibilities in the study of intracellular processes for developing novel methods of diagnostics and complex treatment of various diseases.

\section{Speckle interferometric setup}

We designed and mounted the setup, the general view of which is presented in Figure 1. The optical scheme of the device is presented in Figure 2.

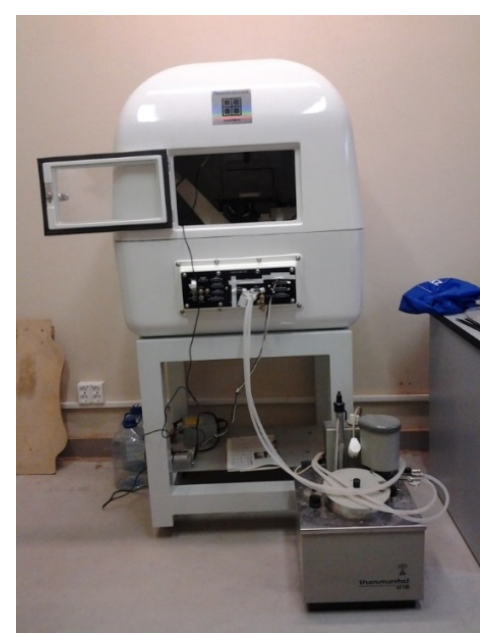

Fig. 1 Photograph of the experimental setup.

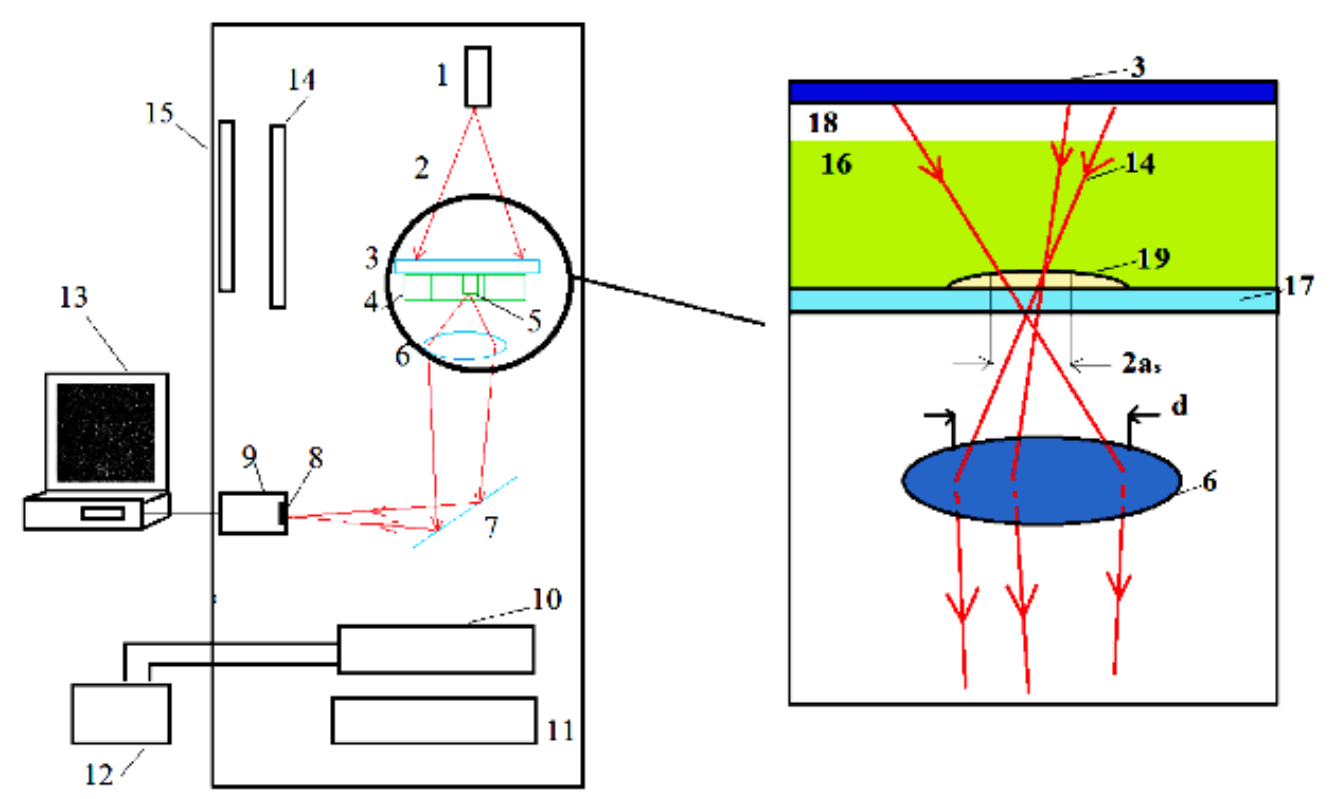

1 - laser, 2 - coherent radiation, 3 - diffuser, 4 - sectioned cuvette, 5 - section with cell culture, 6 - microscope lens, 7 - mirror, 8 - array of photodetectors, 9 - TV camera, 10 - radiator, 11 - fan, 12 - thermostat, 13 - notebook, 14 - thermometer, 15 - window, 16 - nutrient solution, 17 - cuvette bottom, 18 - air medium, 19 - cell; 2as - linear magnification of the microscope, $\mathrm{d}-$ microscope lens aperture diameter.

Fig. 2 Schemes of the optical setup and ray paths.

The authors of Ref. [2] report that the cell viability is observed even under the 300 seconds exposure with the light at the wavelength $660 \mathrm{~nm}$ with the power $20 \mathrm{~mW}$, the beam cross section area being nearly $0.25 \mu \mathrm{m}^{2}$ (the power density $8 \mathrm{~mW} / \mathrm{cm}^{2}$ ). In the present study as the source of light 1 we used the semiconductor laser unit with the wavelength $\lambda=0.65 \mu \mathrm{m}$ and the power $20 \mathrm{~mW}$, the power density being $2.1 \mathrm{~mW} / \mathrm{cm}^{2}$. The radiation from the laser was incident on the diffuser 3 and passed through the cuvette 4 with the studied sample. The cuvette bottom with cells sediment was separated from the diffuser by nearly $10 \mathrm{~mm}$. Then the radiation arrived at the objective 6 with the aperture providing the formation of speckle pattern. In the experiments, we used a monochrome TV camera Videoscan-415/P/KUSB. The camera had an array of photodetectors $6.5 \times 4.8 \mathrm{~mm}$ with $780 \times 582$ pixels, each having the dimensions $8.3 \times 8.3 \mu \mathrm{m}$. The frame repetition rate was up to $25 \mathrm{~Hz}$. The TV camera exposure time was 9 seconds, which exceeded the correlation time of 
radiation intensity (5-8 seconds), corresponding to the most rapid processes [10].

The optimal temperature for the cell culture growth is known to be $36.6 \pm 0.5^{\circ} \mathrm{C}$, and the decrease of the temperature leads to immediate reduction of the cell physiological activity. Therefore, the system was placed in a thermostat 12 . The signals from the TV camera via the USB port were transmitted to the Aspire 3692 WLMi (Acer) notebook 13.

The calibration of the instrument was implemented using a thin plate with the roughness parameter $\mathrm{Ra}$ about $10 \mathrm{~nm}$. The procedure of calibration is rather simple and described in [10].

\subsection{Software}

To enter the image frames we used the «Viewer» software supplied together with the VideoScan camera. The Program has options that allow the recording of individual frames, as well as the movies of speckle dynamics. The frames are recorded in the bmp format. The program allows setting the signal amplification, the frame exposure time, and controlling the rate of the input frames.

Further processing of the experimental data was implemented using the standard packages of Microsoft Excel 2010.

\subsection{Technique of experiment and processing of the results}

The nutrient solution with cells was placed in the cuvette. The cuvette was covered with the diffuser and put into the setup, where the thermostat kept the temperature equal to $36^{\circ} \mathrm{C}$. To measure the temperature in the setup the thermometer was used.

Using the special software, we recorded the values of the parameter $\eta$, characterising the cell metabolic activity and calculated using the formula:

$$
\eta=\frac{\frac{1}{m \cdot n} \sum_{i=0}^{m-1} \sum_{j=0}^{n-1}\left[A_{i, j}-\bar{A}\right]\left[B_{i, j}-\bar{B}\right]}{\sqrt{\frac{1}{m \cdot n} \sum_{i=0}^{m-1} \sum_{j=0}^{n-1}\left[A_{i, j}-\bar{A}\right]^{2}} \cdot \sqrt{\frac{1}{m \cdot n} \sum_{i=0}^{m-1} \sum_{j=0}^{n-1}\left[B_{i, j}-\bar{B}\right]^{2}}}
$$

where $A_{i, j}$ are the signals in the area of $m \times n$ pixels at the initial moment of time, $B_{i, j}$ are the signals from the same area at a different moment of time $\tau, i, j$ are the pixel numbers in the $x$ and $y$ directions, respectively, $\bar{A}$ is the mean values of the signals for the area at the initial moment of time, $\bar{B}$ is the same mean value at the time moment $\tau$.

\subsection{Object of study}

As the object of study, we took the cell cultures L-41 $\mathrm{KD} / 84$. The cell culture L-41 is a cancer culture. The specialists from the Ekaterinburg Research Institute of Virus Infections performed the preparation of biological samples.
According to the certificate of the cell line L-41 $\mathrm{KD} / 84$ [13], the strain of transferred cells G-96 is known to be extracted from the blood of a patient suffering from monocytic leukaemia. In 1966 V.D. Solovyov et al. subjected it to triple impact of large doses of the Koksaki B3 virus to obtain the subline (G-41), specifically resistant to this virus. The clone line L-41 KD/84 was obtained in 1984 in the Laboratory of Cell Cultures, the Ekaterinburg Research Institute of Virus Infections, using the Puck double cloning method with subsequent treatment with antibiotics.

Morphologically the cell line L-41 is a uniform monolayer of distinct epithelium-like polygonal or rounded cells, among which $4-6 \%$ of gigantic cells are permanently present. The cytoplasm is fine-grained. The nuclei are rounded and contain 2-4 nucleoli. The anomalous mitosis forms are up to $6 \%$ and the number of cell with irregular shape of nuclei is up to $8 \%$. The monolayer is formed in 3-5 days from the moment of seeding in the medium, consisting of equal volumes of the "Igla" and "199" media with the addition of $10 \%$ bovine embryo serum. The cell maintenance medium contains the necessary amino acids, vitamins, salts, and glucose.

The culture is highly sensitive to viruses of poliomyelitis, Koksaki B, ECHO-19, human adenoviruses, and the measles virus. The mean cell size is $20-30 \mu \mathrm{m}$.

\section{Results and discussion}

Figure 3 presents a typical speckle pattern observed at a certain fixed moment of time at the computer monitor. One can easily distinguish the cells of the L-41 culture that appeared to be deposited on the substrate during one hour. The frame exposure time was equal to 9 seconds.

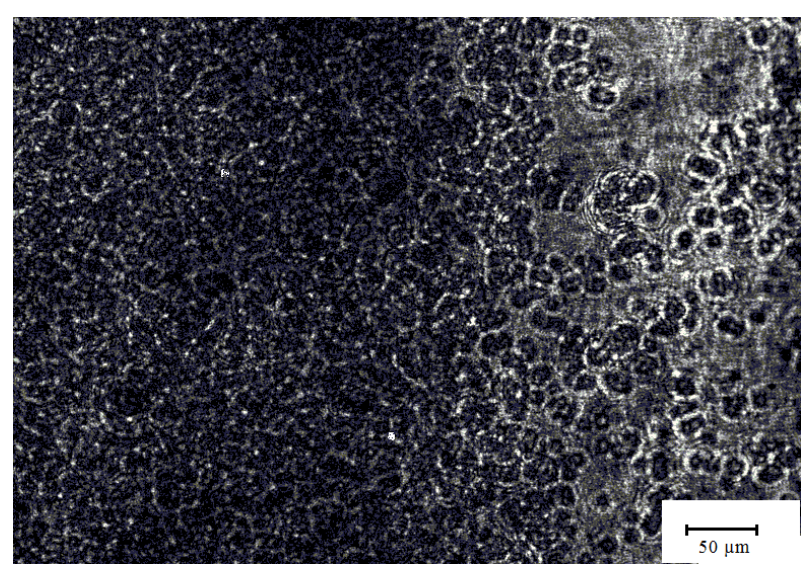

Fig. 3 Speckle pattern for the cell culture L-41.

The performed observations have shown that the cells are not stable. The changes occur both inside each cell and in the entire cell array. We managed to select a few cells, which among others could be considered as visually immobile during an hour. Figure 4 presents a typical dependence $\eta=\eta(t)$ for a stable cell. The value of 
the parameter $\eta$ was found by averaging over the area, the size of which was nearly equal to that of a cell.

For stable cells we determined also the autocorrelation function $\eta=\eta(t)$ for small regions in the cell image. The size of the regions was $5 \times 5$ pixels. Figures 5 and 6 present typical autocorrelation functions of the regions near the cell edge and in the centre of the cell, respectively.

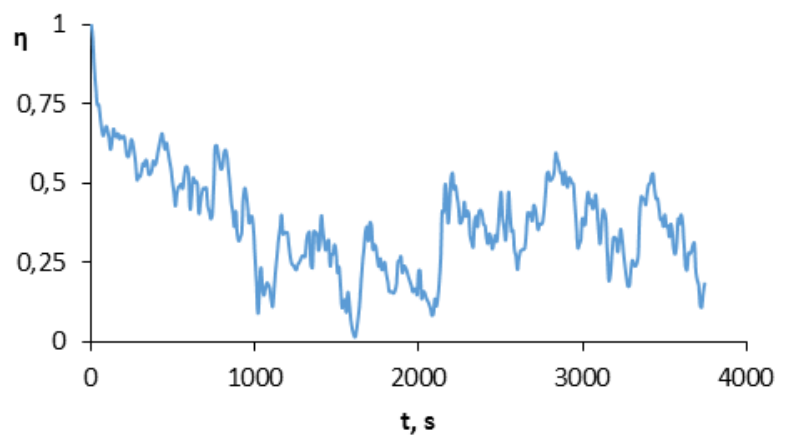

Fig. 4 Typical autocorrelation function for a stable cell.

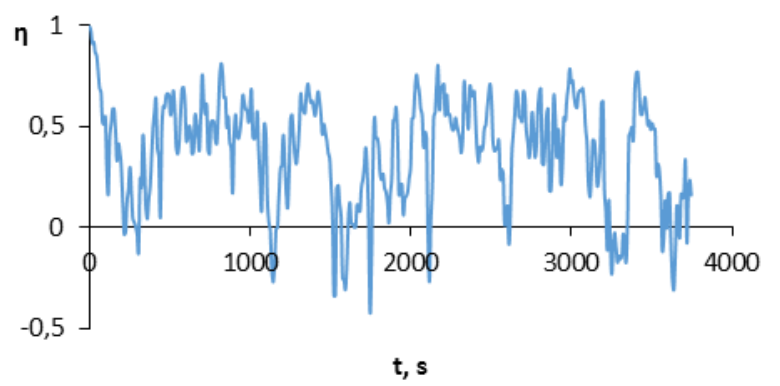

Fig. 5 Typical autocorrelation function for a region inside the stable cell image near the edge.

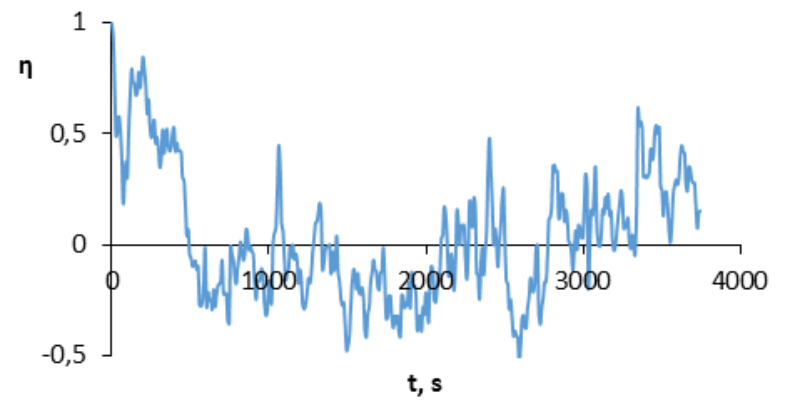

Fig. 6 Typical autocorrelation function for a region inside the stable cell image near the centre.

Figure 7 presents typical autocorrelation functions, corresponding to individual unstable cells. The size of the studied regions was $20 \times 20$ pixels.

The analysis of the obtained data has shown that if the size of the region of averaging is greater of equal to that of a single cell, then for stable cells the autocorrelation function varies within the limits from 0 to 1 , while for unstable cells $\eta$ can take both positive and negative values.

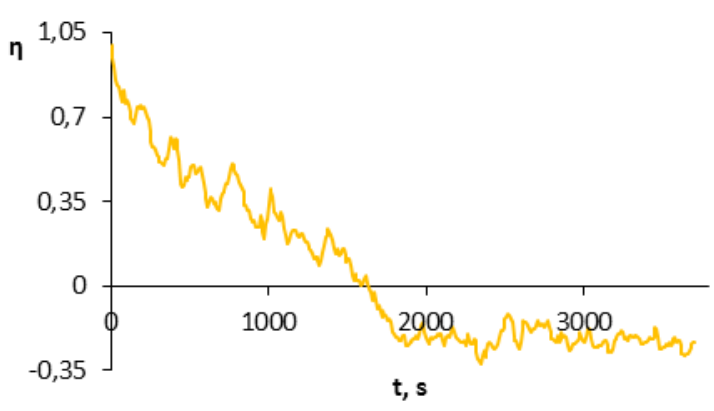

Fig. 7 Typical autocorrelation function of unstable cells.

Using the speckle interferometric setup we also performed the experiment with relatively small number of cultivated cells in the nutrient solution. The object of study was not a monolayer, but individual cells with the typical separation of $2-5 \mu \mathrm{m}$ between them. Figure 8 presents the dependences $\eta=\eta(t)$ for different regions of the cell image and the dependence $\eta=\eta(t)$ for the nutrient solution

As seen from Fig. 8, the value of $\eta$ for the region outside the cell varies within the limits from 0.95 to 0.99 , while for the region inside the cell the variation occurs with the limits from 0.2 to 0.99 .

Thus, from the presented data one can see that the setup and the method allow the distinction of processes that occur in the nutrient solution and in the cells.

The autocorrelation functions plotted above were calculated in small regions in different cell parts, as well as by averaging over the entire cell. For immobile cells, one can see that the correlation coefficient, corresponding to intracellular regions, can take both positive and negative values. However, in the case of a separate stable cell, the values of the correlation coefficients are only positive, and this is an important result. For an unstable cell, the values of the correlation coefficient can be positive and negative in the case of averaging over a single cell. This is probably due to the cell displacements. The clarification of this issue is a subject of further study. The aim of the present analysis was to present new experimental results. In future, the authors plan to analyse the experimental results with the attraction of theory.

In Ref. [11] the formula is presented that relates the correlation coefficient $\eta$ and the root-mean-square deviation of the optical path lengths for pair of waves. According to this formula the obtained value of $\eta=0.99$ corresponds to the root-mean-square deviation of optical paths of the order of $1 \mathrm{~nm}$.

Thus, the authors have shown that the present technique allows the analysis of the variation of the optical path difference of wave pairs, averaged over the region with the size of a few micrometres. The drawback of the methods at present is the averaging of the data over the cell thickness. Therefore, further studies are required on the development of living cell tomography in order to get information on the processes with the depth resolution. Besides that, the method is applicable to relatively slow processes. 


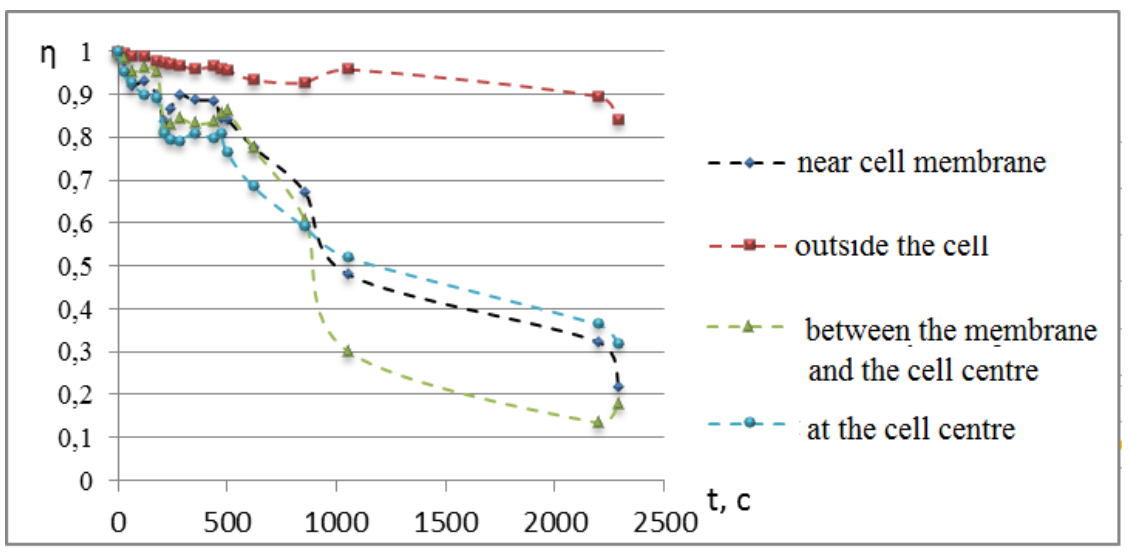

Fig. 8 Dependence $\eta=\eta(t)$ outside and inside the cell.

\section{Conclusion}

Basing on the obtained data, one can draw the following conclusions:

1. The speckle interferometer that allows the determination of speckle dynamics parameters in small regions inside the images of individual cells with overall magnification to $320 \times$ is designed and successfully approved.

2. As a diagnostic parameter, the correlation coefficient for one fragment of speckle pattern taken at two different moments of time is chosen.
3. It is shown that the interferometer allows the registration of the distribution of this parameter within the cell image and beyond it.

4. The difference of the speckle dynamics parameters is revealed between the regions in the cell and outside it, as well as between different regions within one cell image.

The obtained data form a basis for the improvement of the technique. The authors plan to increase the operation rate of the setup and to achieve the temporal resolution of $1 \mu \mathrm{s}$. 